\title{
Investigating the effects of volar wrist cock-up splint and dorsal lock wrist hand orthosis in reducing signs of carpal tunnel syndrome
}

\author{
Behshid Farahmand $^{1}$, Ensieh Pourhosaingholi ${ }^{*} \mathbb{D}^{\mathbb{D}}$, Azam Bagheri $^{1}$
}

Received: 7 Nov 2019

Published: 24 Apr 2021

\section{Abstract}

Background: Carpal tunnel syndrome (CTS) is the most common compressive neuropathy presenting with sharp pain, parenthesis, dysfunction of the hand in coordination and gripping. Splinting is the most common conservative intervention to improve pain and enological symptom of this Syndrome (CTS). With regard to the importance of these interventions and controversies about different designs of splints, the aim of this study was to compare the therapeutic effects of volar wrist cock-up orthosis and dorsal lock wrist hand orthosis on pain, sensory and motor latency in carpal tunnel syndrome.

Methods: In this Randomized controlled trial study, 30 patients diagnosed with mild to moderate CTS were recruited. The subjects were randomly divided into two equal groups. Both groups received one form of splints for three weeks. Before receiving the splints, Electromyography (EMG) and Visual analog scale (VAS) were performed. Then, two different designs of splints were used for a period of three weeks. After that, EMG and Numerical Rating Scale (NRS-11) were repeated to reveal the effects of splints on reducing pain, sensory and motor latency in CTS. Independent $t$ and paired t-tests were done uding SPSS software version 19.0 . Pvalue was set at 0.05 .

Results: All the variables in both groups showed significant improvement. The NRS-11 test was significantly improved in the dorsal lock wrist hand orthosis group $(\mathrm{p}<0.05)$.

Conclusion: This study showed that the use of the dorsal lock wrist hand orthosis for about three weeks was significantly improved pain and neurological symptoms of patients with CTS because of maintaining the wrist in the neutral position. Knowing this fact helps us to design and make a less cumbersome and restrictive splint with an accurate position for the wrist and distal joints.

Keywords: Carpal Tunnel Syndrome, Electromyography, Wrist Splint

Conflicts of Interest: None declared

Funding: None

*This work has been published under CC BY-NC-SA 1.0 license.

Copyright $\subseteq$ Iran University of Medical Sciences

Cite this article as: Farahmand B, Pourhosaingholi E, Bagheri A. Investigating the effects of volar wrist cock-up splint and dorsal lock wrist hand orthosis in reducing signs of carpal tunnel syndrome. Med J Islam Repub Iran. 2021 (24 Apr);35:53. https://doi.org/10.47176/mjiri.35.53

\section{Introduction}

Carpal tunnel syndrome (CTS) is the most common compressive neuropathy in the upper extremity, causes motor and sensory impairment in the median nerve distribution of the hand (1-5). The main symptoms of this disorder are sharp pain, parenthesis, dysfunction of the hand in coordination and gripping $(1,6,7)$ that are often worsened by daily activities or at night $(1,7,8)$. Frequent,

Corresponding author: Dr Ensieh Pourhosaingholi, ensiehpmd@yahoo.com

1. Department of Orthotics \& Prosthetics, School of Rehabilitation, Iran University of Medical Sciences, Tehran, Iran forceful grasping and flexed wrist posture for a long time, or hand-held vibration tools were exposed subjects to CTS $(1-3,7)$. To determine the sensory deficit of CTS, twopoint discrimination test, vibration, and monofilament testing were used $(1,7,8)$. The prevalence of this syndrome was estimated at about $9.2 \%-15.6 \%$ in females and $6 \%-11.3 \%$ in males $(2,6,7)$. Age and gender are related

\section{$\uparrow$ What is "already known" in this topic:}

Several techniques have been introduced for the treatment of Carpal Tunnel Syndrome (CTS). Wrist splints reduce pain and other symptoms of CTS.

\section{$\rightarrow$ What this article adds:}

The result of this study showed that the use of the dorsal design cock-up splint relieves signs and symptoms of CTS. The results help us to make a better decision for the treatment for CTS. 
to the incidence of CTS (7).

For motor and sensory nerve evaluation of this syndrome, electromyography (EMG) and nerve conduction velocities were used to diagnosis and define the severity of CTS $(2,4,6,9-13)$. The most common conservative non-surgical intervention of mild to moderate CTS in rehabilitation is to use night (static) splints. That seems to reduce pain and other symptoms of CTS $(6,12,14)$, with rest and evoked with activity and reduced carpal tunnel pressure $(5,6)$. These devices immobilize the wrist in the neutral position (less until 10 degrees) $(6,11,12,14)$. This position seems to reduce pain and other symptoms of CTS by maximizing carpal tunnel volume and minimizing the pressure on the median nerve $(2,4,13,14)$.

Several studies have demonstrated the efficacy of wrist splints in relieving the signs and symptoms of CTS; however, the chosen angle of immobilization, selected design and effects of these designs on pain and symptoms of CTS were confusing $(1,4,13-15)$. The rationale for these splint designs was based on the concept of positioning the fingers, which affected the carpal tunnel size. These designs alter the shape of the carpal tunnel by moving the lumbricals more distal out of the carpal tunnel and decrease pressure on the median nerve. The results of some researches were indicated that the measurements of the electromyography/nerve conduction study (EMG/NCS) provide information about the probability effects of splinting $(1,2,15$, 16).

Nonetheless, a lot of debates have always existed about the best designs and positions for splinting the hand and wrist (7). Furthermore, no study has been performed on different aspects of splinting like volar or dorsal designs and wrist position in the splint.

In the current study, two designs of splint (one volar and one dorsal) that keep the wrist in a neutral position (0-5 degree) were used.

Therefore, the aim of this study was to compare the therapeutic effects of volar wrist cock-up orthosis and dorsal lock wristhand orthosis on pain, sensory and motor latency in carpal tunnel syndrome.

\section{Methods}

This interventional study was designed as a randomized controlled trial to explore the effects of two types of splints on the symptoms of CTS. This study was approved by the ethics committee of Isfahan University of Medical Sciences. The protocol has been registered in the Iranian Registry of Clinical Trials (registration code: IRCT1389903224157N1).

Thirty patients (18 females, 12 male) with a mean age of $58.2( \pm 12.1)$ years and mean weight of $87.6( \pm 5.5) \mathrm{Kg}$ were recruited and enrolled in this study. All the subjects in the treatment group were right-handed. The mean age of the control group was $45.76( \pm 11.6)$ years and the mean age of the treatment group was $47.42( \pm 6.1)$ years. CTS diagnosis was confirmed with physical examination and nerve conduction test of the median nerve according to the American Association of Electro-diagnostic Medicine guidelines (mild to moderate cases) (17). Once recruited and selected for participation, patients were ran- domly assigned to either the two equal groups and received randomly one of the splints for a period of three weeks. Every patient had EMG and NAS tests before starting the treatment $(19,20)$.

All participants received trial information and provided written informed consent. All patients signed for informed consent prior to the start of the study and the study protocol was approved by the Medical Ethics Committee.

Patients with mild to moderate CTS (9) were qualified for participation based on the inclusion criteria of 1) having positive clinical examination finding consistent with CTS (Tinel's sign or Phalen maneuver), including 2) having reported night time pain, numbness, and tingling for at least 12 weeks, 3) patients with sensory conduction latency with a peak greater than $4.00 \mathrm{~ms}$ using ring pick up on the volar surface of the index finger measured $14 \mathrm{~cm}$ from stimulation across the wrist at the median nerve, 3) patients with motor conduction latency of greater than 4.2 ms measured with disc pick up on the abductor pollicis brevis muscle $8 \mathrm{~cm}$ from the stimulation site across the wrist at the median nerve.

Patients with a history of hand surgery, systemic diseases, those had a steroid injection into the carpal canal within the previous 6 months, pregnancy, wrist-fracture histories, a lack of cooperation, and a lack of nerve-conduction velocity were excluded $(9,18,19)$. Furthermore, patients who presented with neuropathy or radiculopathy, thenar atrophy as a symptom of severity, patients with a ganglion cyst of the wrist, lack of cognition, history of hand surgery, sensory conduction latency to peak less than $3.7 \mathrm{~ms}$ when measured with ring pick up on the volar surface of the index finger measured $14 \mathrm{~cm}$ from stimulation across the wrist at the median nerve were excluded $(18,19)$.

The electromyography test was carried out by a "MEDLEC SYNERGY VIASIS 3/05" electromyography device with two 6-mm felt tip bar electrodes as the stimulators and recorders (with a diameter of pads $23 \mathrm{~mm}$ apart). The antidromic sensory nerve action potentials evoked at the wrist were recorded from the middle finger. Standard distances $(7 \mathrm{~cm}$ from the recorder at mid-palm and $14 \mathrm{~cm}$ from the recorder at the wrist) were kept between the stimulator and the recorder electrodes. The sensory nerve conduction velocity was recorded in meters per second and the sensory distal latency was recorded in milliseconds.

After giving written informed consent and a baseline assessment, participants of the current study were divided into two groups (those who wore a neutral wrist splint in a form of short cock up and those who wore a neutral wrist in a form of dorsal lock using a randomized allocation between participants $(21,22)$.

The patients were taught how to use the splint correctly. The initial evaluations were repeated after three weeks for both groups and data was gathered.

Two types of prefabricated splints (volar cock up and dorsal lock) were used in the current study and all patients received these prefabricated splints after accurate measurement of hand and wrist for better fitting (Fig. 1) (20, 21).

Dorsal lock splint puts the wrist in neutral position of 
$\left(0^{\circ}-5\right)(20)$ and sets the MCP joints positioned between $0^{\circ}$ and $10^{\circ}$ of flexion and PIP in 20-30 degree flexion DIP and the MCP joint of the thumb was free to move in all direction (Fig. 1) (21-23).

In volar wrist cock-up, the splint just embraces the wrist in neutral position of $\left(0^{\circ}-5\right)$ and ended just proximal to the MCP; as MCP and PIP joints are free from restriction, and set the MCP joints positioned between $0^{\circ}$ and 5 degrees of flexion $(20,21)$. The distal palmar crease and proximal palmar crease of the hand must be fully cleared when fabricating a volar-cock up splint. Then non elastic Velcro closures were used to increase the fitness of splints and reduce relative movement between the hand and wrist. Patients were instructed to wear the splint during the night for at least 3 weeks and during the day only if they wished to. There were no restrictions for the patients in their work or normal daily activities. EMG and the Numeric Rating Scale (NRS-11) were accomplished (24).

After 3 weeks of using splints, the EMG and the Numeric Rating Scale (NRS-11) test were repeated. According to some study we used two main characteristics of EMG measurement, sensory latency difference greater than $0.4 \mathrm{~m} / \mathrm{sec}$ or distal motor latency (DML) greater than $4.2 \mathrm{~m} / \mathrm{sec}$ as CTS. We also repeated the EMG measurement to know about the effect of splint on sensory latency difference and distal motor latency.

Then, all patients were given the NAS (Numeric Analog Scale) which has been validated as a reliable and reproducible outcome to measure the severity of symptoms and functional status in CTS (1).

\section{Numeric Analog Scale}

The Numeric Rating Scale (NRS-11) is an 11-point scale for patients' self-reporting of pain. It is for adults and children older that 10 years of age (Table 1) (22-28).

Patients reported their maximum pain at the start of the examination and at the end of 3 weeks after using splints

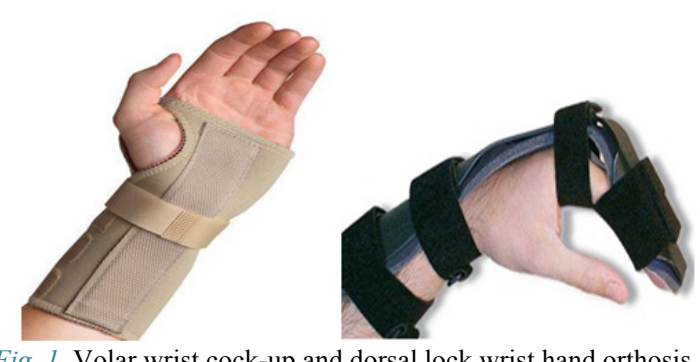

Fig. 1. Volar wrist cock-up and dorsal lock wrist hand orthosis

Table 1. The Numeric Rating Scale (NRS-11) with 11-point scale for pain

\begin{tabular}{lc}
\hline Rating & Pain Level \\
\hline 0 & No Pain \\
$1-3$ & Mild Pain (nagging, annoying, interfering little with ADLs) \\
$4-6$ & Moderate Pain (interferes significantly with ADLs) \\
$7-11$ & Severe Pain (disabling; unable to perform ADLs) \\
\hline
\end{tabular}

(24).

\section{Statistical analysis}

All statistical analysis were performed using SPSS Statistic 19.0, (SPSS, Inc., Chicago, IL, USA) with a P-value set at 0.05 . The data were tested for normal distribution using the Kolmogorov-Smirnov test. The test of normality, independent $\mathrm{t}$ and paired $\mathrm{t}$-tests with a $95 \%$ confidence interval $(\alpha=0.05)$.

\section{Results}

The results of this study showed a significant difference between using the two splints (dorsal lock/short cock up) and without splint $(\mathrm{p}<0.05)$ (Tables 2 and 3$)$.

The results of this study showed a significant difference between using the two splints of dorsal lock and short cock up $(\mathrm{p}<0.05)$ (Table 4$)$.

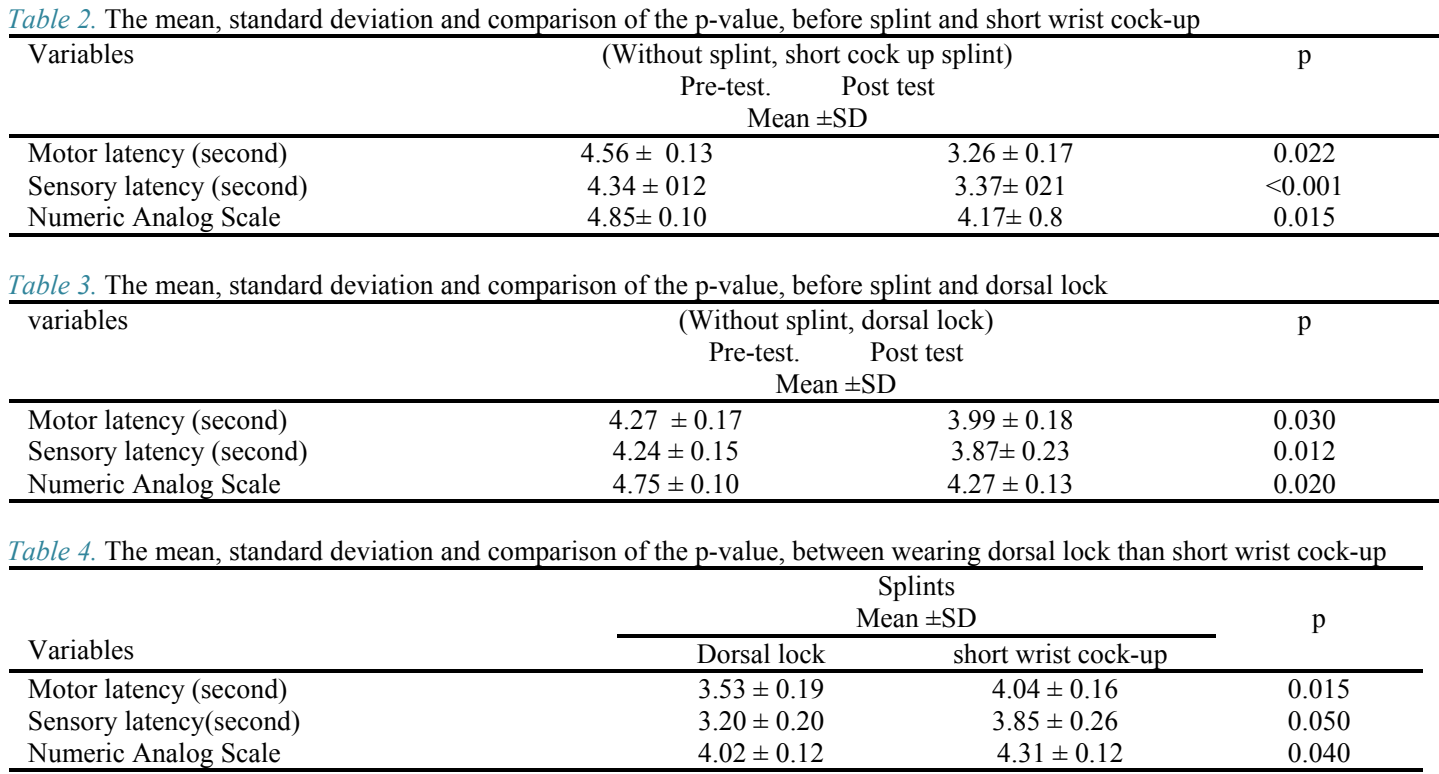




\section{Discussion}

The aim of the current study was to compare the effectiveness of two designs of splints in reducing symptoms included pain of carpal tunnel syndrome (CTS).

The results of current study showed that the use of the two types of splints has a significant effect on the pain and symptom of CTS. There has been no similar study on the comparison of different kinds of splints; $(28,29)$ some studies were found in this study with similarities to this research $(6,9,11,20,22,25,26)$. This study addresses the need for different types of splints as modalities that may treat the signs and symptoms of CTS $(6,9,11,20,22,25$, 26). However, selected angles for wrist immobilization are varied in different designs of splints and in our experience, some physicians prescribe splints without attention to the wrist angel; this leads to disappointment of the patients regarding the effects of splint $(26,27)$.

Moreover, there are a variety of splint designs from various manufactures which reduces the pain and other signs and symptoms of CTS (27). Some of these designs hold the wrist near functional angel (more than 20) which seems to increase inter-carpal pressure $(27,28)(18,19)$.

These splints not only assist these patients with CTS to perform ADL, but also reduce the symptoms and the pain of their upper extremity (16).

The motor latency and the sensory latency were significantly improved with dorsal lock compared to short cock up $(\mathrm{p}<0.05)$.

This study briefly compared the two designs of splint which has an effect in reducing pain of CTS. However dorsal lock seems to improve the pain more significantly than the short cock up.

Some authors believe that special designs may reduce inter carpal pressure and symptoms of carpal tunnel; however, according to our study dorsal design splint significantly improved the symptom and pain of CTS compared to the volar one $(27,28)$.

Some authors believe that splint should be removed as quickly as possible, unless necessary to accomplish a short-term task (29-31). We do not agree that using splint increases the inter-carpal pressure or presses the muscles, but further studies are needed.

In this regard, Walker et al. (14) mentioned that part time use of splints in night had a more positive effects on functional activities than full-time use. Furthermore, a full-time use of splints was not preferred by patients and most of the time, they rejected splints.(5).

Finally, it seems that EMG, as a diagnostic tool, may be a useful tool to categorize different levels of tunnel injury, including cases that were improved with splint and severe cases candidate for surgery (29).

Our study does not show long-term results. Therefore, we could not predict long-term effect of splints $(28,29)$.

There are some limitations in this study. First, this study sample size was relatively small. Moreover, in our patients, wrist splints were worn basically at night, and also during the daytime in some patients according to their symptoms. In addition, this study did not have a noninterventional control group.

\section{Conclusion}

This study showed that the use of the wrist hand splints for three weeks could have a significant effect on the pain and symptom of patients with CTS. Furthermore, by maintaining the wrist in neutral position that keeps the inter-tunnel pressure at the lowest level or using dorsal design compared to circumferential or palmar splints may improve the result of this intervention. Knowing this fact helps us to design and make less cumbersome and restrictive splint with an accurate position for the wrist and distal joints.

\section{Conflict of Interests}

The authors declare that they have no competing interests.

\section{References}

1. Sardana V, Ojha P. Carpal Tunnel Syndrome: Current Review.

2. Sakly G, Affes Z, Ben Dhia S, Trabelsi A, Ben Cheikh R, Gammoudi $\mathrm{N}$, et al. The Impact of Carpal Tunnel Syndrome on Ulnar Nerve: An Electro-Clinical Study. J Neurol Neurosci. 2016;7(5).

3. Jablecki CK, Andary MT, So YT, Wilkins DE, Williams FH. Literature review of the usefulness of nerve conduction studies and electromyography for the evaluation of patients with carpal tunnel syndrome. Muscle Nerve. 1993;16(12):p. 1392-1414.

4. Visser LH, Smidt MH, Lee ML. High-resolution sonography versus EMG in the diagnosis of carpal tunnel syndrome. J Neurol Neurosurg Psychiatry. 2008;79(1):63-67.

5. Armstrong TJ, Chaffin DB. Some biomechanical aspects of the carpal tunnel. J Biomec. 1979;12(7):567-570.

6. Stewart B, Lancaster G, Lawson J, Williams K, Daly J. Validation of the Alder Hey triage pain score. Arch Dis Child. 2004;89(7):625630 .

7. von Baeyer CL, Spagrud LJ. Systematic review of observational (behavioral) measures of pain for children and adolescents aged 3 to 18 years. Pain. 2007;127(1):140-150.

8. Feldt, K.S., The checklist of nonverbal pain indicators (CNPI). Pain Manag Nurs. 2000;1(1):13-21.

9. Stinson JN, Kavanagh T, Yamada J, Gill N, Stevens B. Systematic review of the psychometric properties, interpretability and feasibility of self-report pain intensity measures for use in clinical trials in children and adolescents. Pain. 2006;125(1):143-157.

10. Cleeland CS, Ryan K. The Brief Pain Inventory. Available online: https://www.mdanderson.org/education and research/departmentsprograms-and-labs/departments-and-divisions/symptom-research/ symptomassessment-tools/BPI_UserGuide. pdf (accessed on 1 March 2016), 1994.

11. Hicks CL, von Baeyer CL, Spafford PA, van Korlaar I, Goodenough B. The Faces Pain Scale-Revised: toward a common metric in pediatric pain measurement. Pain. 2001;93(2):173-183.

12. Stratford PW, Binkley JM, Riddle DL, Guyatt GH. Sensitivity to change of the Roland-Morris back pain questionnaire: part 1. Phys Ther. 1998;78(11):1186.

13. Premoselli S, Sioli P, Grossi A, Cerri C. Neutral wrist splinting in carpal tunnel syndrome: a 3-and 6-months clinical and neurophysiologic follow-up evaluation of night-only splint therapy. Euro Medicophys. 2006;42(2):121-126.

14. Walker WC, Metzler M, Cifu DX, Swartz Z. Neutral wrist splinting in carpal tunnel syndrome: a comparison of night-only versus full-time wear instructions. Arch Phys Med Rehab. 2000;81(4):424-429.

15. Wali A, Ahmed R, Khan S. Electrophysiological evidence of the Riche-Cannieu anastomosis in the hand and its diagnostic implications; 2 case reports. CNP J. 2017;2:8-11.

16. Ozguler A, Guéguen A, Leclerc A, Landre MF, Piciotti M, Le Gall $\mathrm{S}$, et al. Using the Dallas Pain Questionnaire to classify individuals with low back pain in a working population. Spine. 2002;27(16):17831789.

17. Marmor MF, Tan F. Central serous chorioretinopathy: bilateral multifocal electroretinographic abnormalities. Arch Ophthalmo J. 1999;117(2):184-188.

18. Jablecki C, Andary MT, Floeter MK, Miller RG, Quartly CA, 
Vennix M J, et al. Practice parameter: electrodiagnostic studies in carpal tunnel syndrome report of the American Association of Electrodiagnostic Medicine, American Academy of Neurology, and the American Academy of Physical Medicine and Rehabilitation. Neurology. 2002;58(11):1589-1592

19. Kimura J. Electrodiagnosis in diseases of nerve and muscle: principles and practice 2001: Oxford university press.

20. Page MJ, Massy-Westropp N, O'Connor D, Pitt V. Splinting for carpal tunnel syndrome. Cochrane Library. 2012.

21. Zinnuroglu M, Baspinar M, Beyazova M. Carpal lock and the volarsupporting orthosis in mild and moderate carpal tunnel syndrome. Am J Phys Med Rehabili. 2010;89(9):759-764.

22. Gerritsen AA, de Vet HCW, Scholten RJPM, Bertelsmann FW, de Krom MCTFM, Bouter LM. Splinting vs surgery in the treatment of carpal tunnel syndrome: a randomized controlled trial. JAMA. 2002;288(10):1245-1251.

23. Werner RA, Andary M. Electrodiagnostic evaluation of carpal tunnel syndrome. Muscle Nerve. 2011;44(4):597-607.

24. von Baeyer CL, Spagrud LJ, McCormick JC, Choo E, Neville K, Connelly MA, et al. Two new datasets supporting use of the Numerical Rating Scale (NRS-11) for children's self-reports of pain intensity. Pain. 2009;143(3):223-227.

25. Guyette T, Wilgis E. Timing of improvement after carpal tunnel release. J Surg Ortho Adv.2003;13(4):206-209.

26. Nobuta S, Sato K, Nakagawa T, Hatori M, Itoi E. Effects of wrist splinting for carpal tunnel syndrome and motor nerve conduction measurements. Ups J Med Sci. 2008;113(2):181-192.

27. Mishra S, Prabhakar S, Lal V, Modi M, Das ChP, Khurana D. Efficacy of splinting and oral steroids in the treatment of carpal tunnel syndrome: a prospective randomized clinical and electrophysiological study. Neurol India. 2006;54(3):286.

28. Burke FD, Wilgis EFS, Dubin NH, Bradley MJ, Sinha Sh. Relationship between the duration and severity of symptoms and the outcome of carpal tunnel surgery. J Hand Surg. 2006 Nov;31(9):147882.

29. Özgen M, Güngen G, Sarsan A, Ardıç F, Calıskan S, Sabir N, et al. Determination of the position on which the median nerve compression is at the lowest in carpal tunnel syndrome and clinical effectiveness of custom splint application. Rheumatol Int. 2011;31(8):1031-1036.

30. Manente G, Melchionda D, Staniscia T, D'Archivio C, Mazzone V, Macarini L. Changes in the carpal tunnel while wearing the Manu ${ }^{\circledR}$ soft hand brace: a sonographic study. J Hand Surg Eur Vol. 2013;38(1):57-60.

31. Schmid AB, Elliott JM, Strudwick MW, Little M, Coppieters MW. Effect of splinting and exercise on intraneural edema of the median nerve in carpal tunnel syndrome - an MRI study to reveal therapeutic mechanisms. J Orthop Res. 2012;30:343-1350. 